\title{
Altered Calreticulin expression in human colon cancer: Maintenance of Calreticulin expression is associated with mucinous differentiation
}

\author{
C. TOQUET ${ }^{1-3}$, A. JARRY ${ }^{1}$, C. BOU-HANNA ${ }^{1,2}$, K. BACH $^{1-3}$, \\ M.G. DENIS ${ }^{1-3}$, J.F. MOSNIER ${ }^{1-3}$ and C.L. LABOISSE ${ }^{1-3}$ \\ ${ }^{1}$ INSERM, U539; ${ }^{2}$ Université de Nantes, Faculté de Médecine de Nantes; ${ }^{3} \mathrm{CHU}$ de Nantes, 44035 Nantes, France
}

Received September 4, 2006; Accepted November 24, 2006

\begin{abstract}
Calreticulin is an endoplasmic reticulum luminal calcium-binding chaperone involved in various cellular functions and is a ligand for the scavenger receptor CD91. Recent studies, based on proteomic approaches on whole tissue samples containing both neoplastic and non-neoplastic cells, have shown alterations of Calreticulin expression in colon carcinomas, albeit with divergent results. The aims of this study were: 1) to assess the expression of Calreticulin and its receptor CD91 in 58 human colon adenocarcinomas, compared with paired normal mucosa, using a semi-quantitative immunohistochemical analysis, and 2) to examine associations between the tumour phenotypic features, and Calreticulin and/or CD91 expressions. Calreticulin expression was down-regulated in $51.7 \%$ human colon adenocarcinomas. Accordingly, quantitative immunoblot analysis showed that Calreticulin expression was significantly lower in human colonic cancer cell lines than in preparations of isolated human normal colonic epithelial cells. CD91 was co-expressed with Calreticulin in both normal colonic epithelial cells and pericryptic myofibroblasts. Calreticulin and CD91, that characterize the 'amateur phagocyte' function of epithelial cells, were both downregulated in $48 \%$ of adenocarcinomas. Finally, Calreticulin expression was significantly associated with the mucinous differentiation of the tumour. Collectively, these results show that Calreticulin is likely to play a pivotal role in the differentiation of human colonic adenocarcinomas.
\end{abstract}

\section{Introduction}

Calreticulin, an ancient and highly conserved protein, is an ER luminal calcium-binding chaperone involved in many cellular functions such as regulation of calcium homeostasis and

Correspondence to: Professor C.L. Laboisse, INSERM U539, Faculté de Médecine, 1 Rue Gaston Veil, 44035 Nantes, France E-mail: u539@nantes.inserm.fr

Key words: Calreticulin, CD91, colon cancer, mucinous differentiation calcium-dependent pathways $(1,2)$, lectin-like chaperone activity (3), modulation of cell adhesion (4-6), and modulation of steroid-sensitive gene expression (7). In addition, Calreticulin appears to play an important role in the immune system, since it is involved in the assembly of major histocompatibility complex class I proteins, necessary for class I antigen presentation (8-11). Calreticulin also plays a role in the removal of apoptotic bodies and in the immunomodulation via its receptor CD91, known as an endocytic scavenger receptor expressed by antigen-presenting cells (12-17).

A proteomic study in mice, based on the comparison of colonic epithelial cells from normal and MIN mice (i.e. mice with an APC gene mutation developing numerous intestinal adenomas), shows a down-regulation of Calreticulin expression in MIN adenomas (18). Two other studies, comparing the expression of Calreticulin in human colon adenocarcinomas to that of normal epithelium by proteomic analysis on whole tissue samples containing both neoplastic and non-neoplastic cells, show divergent results i.e. an up-regulation (19) or a down-regulation (20) of Calreticulin in human colon adenocarcinomas. Collectively, these findings suggest that Calreticulin regulation is an early event and could participate in colon carcinogenesis. However, up to now, the comparative expression of Calreticulin in neoplastic and normal paired colonic epithelial cells by immunohistochemistry has not been addressed. This is an important point knowing that Calreticulin may be found not only in epithelial cells but also in the tumour stromal reaction.

The aims of this study were: 1) to assess by immunohistochemistry Calreticulin expression in a series of 58 human colon adenocarcinomas compared with paired normal mucosa, 2) to compare Calreticulin expression in colon adenocarcinomas with that of its receptor CD91, and 3) to examine possible associations between the tumour phenotypic features i.e. tumour localisation, TNM staging, histological grading, presence of a mucinous differentiation, presence of a lymphoid stromal reaction, and Calreticulin and/or CD91 expressions.

\section{Materials and methods}

Tumor samples. Fifty-eight consecutive patients with primary adenocarcinoma treated at the Department of Surgery (Centre Hospitalier Universitaire of Nantes) were included in the study. 

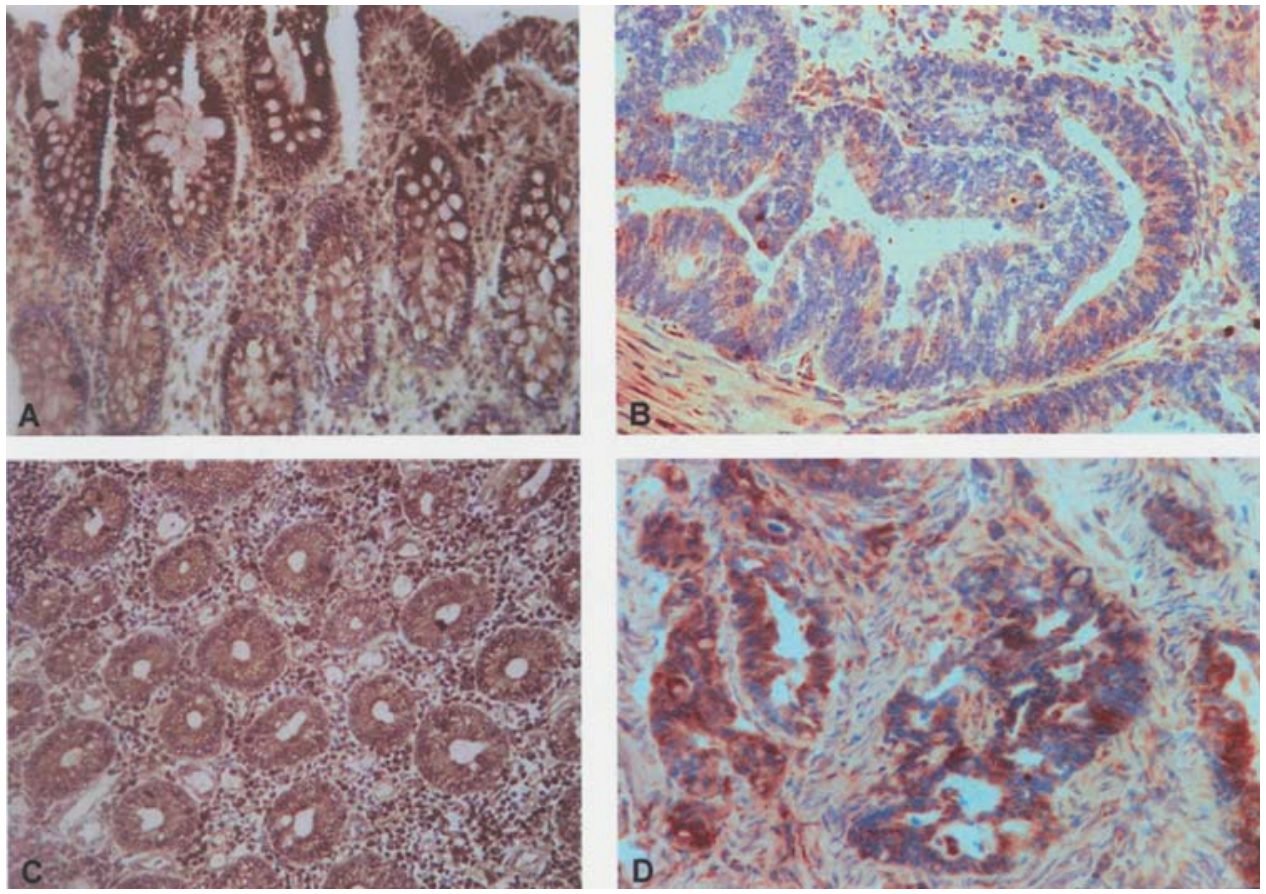

Figure 1. Calreticulin immunohistochemistry in human colonic adenocarcinomas compared with normal paired epithelium. Frozen sections of human adenocarcinomas and normal paired epithelium were stained with the polyclonal anti-Calreticulin antibody and counterstained with hematoxylin. In normal colonic mucosa, Calreticulin was expressed by colonic epithelial cells all along the crypt and also by lamina propria cells (A and C). In colonic adenocarcinomas, Calreticulin expression in neoplastic cells was either down-regulated (B) or similar (D) to that of normal paired epithelium. Calreticulin was also expressed in the stroma of colon carcinoma. Original magnification x 200 .

There were 38 men and 20 women with a median age of 72.5 years (mean 70.4; range 35-87). Patients condition was assessed according to the system of staging primary tumour/ regional lymph nodes/distant metastasis (TNM) described in the AJCC Cancer Manual. The World Health Organisation Classification of tumours was used to determine histological classification. The 58 patients were classified into the TNM stages as follows: stage 1,2 patients; stage 2,33 patients; stage 3, 14 patients; and stage 4, 9 patients. Thirty-six tumors originated in the right colon, 22 in the left colon. None of the patients underwent radiotherapy or chemotherapy before surgery. The tumours, histologically classified according to the WHO classification, were divided into 44 'classic' adenocarcinomas and 14 mucinous adenocarcinomas. 'Classic' adenocarcinomas are usually gland-forming and classified as well, moderately or poorly differenciated adenocarcinomas. They display no mucinous differentiation. Mucinous adenocarcinomas are characterised by pools of extracellular mucins that contain malignant epithelium as acinar structures, strips of cells or single cells, and representing $>50 \%$ of the lesion. Finally, the lymphoid stromal reaction within the tumour was graded as $0,+,++$.

For the 58 patients studied, snap frozen tumor samples and paired normal colonic tissue taken at a $10 \mathrm{~cm}$ distance from the tumour, were available, collected by the Biobank of Institut Régional du Cancer Nantes Atlantique, according to the guidelines of the French Ethics Committee for Research on human tissues.

Immunohistochemical studies. An immunoperoxidase technique was performed by using a streptavidin-biotin peroxidase method on acetone-fixed frozen sections, according to the manufacturer's instructions (LSAB kit, Dako Cytomation, Trappes, France). The primary antibodies used were Calreticulin (1:800; Stressgen, Ann Arbor, MI, USA) and CD91 (1:300; Dako Cytomation). The chromogen used was DAB (3,3'-diaminobenzidine tetrahydrochloride) and tissue sections were counterstained with hematoxylin. Appropriate negative controls (omission of the primary antibody) were used throughout.

An immunolocalisation of Calreticulin and CD91 expression was performed, as well as a semi-quantitative evaluation comparing the expression of tumour cells with that of the paired normal colonic epithelium. An assessment of the number of positive cells in the tumour, as well as the intensity of the cytoplasmic staining was performed. When at least $50 \%$ of tumour cells showed a very low staining or scored negative, the tumour was considered as down-regulated for Calreticulin or CD91 expression relative to the paired normal epithelium.

For co-expression studies, immunofluorescence followed by confocal microscopy was performed on 4 cases of colon cancer and paired normal epithelium. The following doublestaining combinations were performed on $8-\mu \mathrm{m}$ paraformaldehyde-fixed frozen sections: Calreticulin (rabbit, $1: 800) / C D 91$ (mouse, 1:200); $\alpha$-smooth muscle actin ( $\alpha$-SMA, rabbit, 1:400, Abcam, Oxon, UK)/CD91 (mouse, 1:200). After $1 \mathrm{~h}$ of incubation with the primary antibodies, sections were incubated with a mixture of alexa fluor 488-conjugated goat anti-mouse antibody and alexa fluor 568-conjugated goat anti-rabbit antibodies (1:200, Molecular Probes, Eugene, OR, USA). Nuclear staining was performed with TOPRO-3 (1 $\mu \mathrm{M}$, 
Molecular Probes). Imaging was performed on a Leica TCS-SP confocal laser scanning microscope (Leica, Heidelberg, Germany) as previously described (21). Quantification of the overlay of labeling was carried out using the MetaMorph software, as previously described (22).

Isolation of human normal colonic epithelial cells. Human colonic epithelial cells were isolated from histologically normal colon taken at a $10 \mathrm{~cm}$ distance from the tumor of surgical resections for colon cancer using a non-enzymatic dissociation technique as described previously (23). Preparations of colonocytes were devoid of contamination by immune cells (23).

Human colonic cell lines. The following human colonic cancer cell lines were used: the undifferentiated HT29 cell line (24) and its differentiated clonal derivatives HT29-Cl.16E, HT29Cl.19A (25), as well as SW1116, SW480, SW620 (26) and Colo 320 (27). These cell lines were cultured in DMEM (InVitrogen, Cergy Pontoise, France) supplemented with $10 \%$ fetal bovine serum (FBS, Invitrogen) and used at postconfluency or until full differentiation.

Immunoblot analysis. For total protein extraction, human normal colonocytes as well as the various cell lines were lysed in RIPA buffer supplemented with protease inhibitors, and centrifuged. Proteins $(5 \mu \mathrm{g})$ were separated by electrophoresis on $10 \%$ polyacrylamide gel (Bio-Rad, Hercules, CA, USA) and transferred onto polyvinylidene difluoride membranes (PVDF, Invitrogen). After blocking, membranes were incubated with a mixture of rabbit polyclonal antibodies against Calreticulin (1:20,000, StressGen) and mouse monoclonal antibodies against $B$-actin $(1: 10,000$, Sigma, St. Louis, MO, USA), and then with a mixture of alkaline phosphataseconjugated anti-rabbit (1:10,000; Amersham Biosciences, Piscataway, NJ, USA) and anti-mouse antibodies (1:2000, Sigma). Immunoreactive proteins were detected with a fluorescence scanner (Storm, Amersham) using ECF substrate according to the manufacturer's instructions (Amersham). Quantification was performed using the Image Quant software (Amersham), and results were expressed as the ratio of Calreticulin/ß-actin.

Statistical analyses. Statistical analyses were performed with GraphPad Prism version 4.0 (GraphPad software Inc., San Diego, CA, USA). Mann-Withney U test was used to compare Calreticulin/ß-actin levels between cancer cell lines and preparations of normal colonic epithelial cells.

Fisher's exact method was used to test the associations between CD91 and/or Calreticulin expressions and one of the following phenotypic features of the tumours: localisation (right or left colon), TNM staging, presence of a mucinous component, presence of a lymphoid stromal reaction. A twotailed probability of 0.05 was accepted as statistically significant.

\section{Results}

Down-regulation of Calreticulin expression in human colonic adenocarcinomas compared with paired normal epithelium.
In normal colonic mucosa, Calreticulin was expressed by colonic epithelial cells all along the crypt and also by lamina propria mononuclear cells, endothelial cells, fibroblasts and muscularis mucosa smooth muscle cells. All these cells displayed a strong cytoplasmic staining. Epithelial cells also displayed a membrane staining (Fig. 1A and C).

In 58 cases of adenocarcinoma, Calreticulin immunostaining was compared with paired normal colonic mucosa at distance from the tumour using a semi-quantitative evaluation. Calreticulin was found to be down-regulated in 30 out of the 58 cases $(51.7 \%)$ (Fig. 1B). In the majority of the downregulated cases, nearly all tumour cells scored negative or very weakly positive. In a few tumours considered as downregulated, the expression of Calreticulin was more heterogeneous: the majority of tumour lobules scored negative, but a few lobules, representing 10-30\% of tumour cells, displayed a Calreticulin expression similar to that of the normal paired epithelium. In the other 28 cases (48.3\%), the intensity of Calreticulin staining was homogeneous and similar to that of the normal paired epithelium (Fig. 1D). In most cases, the stromal reaction within the tumour, i.e. fibroblasts, endothelial cells, inflammatory cells, smooth muscle cells, strongly expressed Calreticulin.

Down-regulation of Calreticulin expression in colonic cancer cell lines compared with normal isolated colonic epithelial cells. To fully explore Calreticulin expression in human normal isolated colonic epithelial cells and in several human colonic cancer cell lines, quantitative immunoblots using antiCalreticulin and anti- $\beta$-actin antibodies were performed in triplicate on four preparations of normal colonic epithelial cells and seven colonic cancer cell lines. Calreticulin antibody detected a strong unique band at $\sim 60 \mathrm{kDa}$ (Fig. 2A). Fig. 2A, which illustrates a representative immunoblot, shows that Calreticulin expression was higher in isolated colonic epithelial cells than in the various cancer cell lines tested. Quantification of Calreticulin and B-actin was performed on several immunoblots using the Image Quant software for each sample (Fig. 2B). A significant down-regulation of Calreticulin/ $\beta$-actin ratio $(60 \%)$ was observed in the seven colonic cancer cell lines tested $(0.67 \pm 0.05)$, compared with the four isolated colonic epithelial cell preparations $(1.14 \pm 0.05) ; \mathrm{p}=0.0001)$.

Down-regulation of CD91 expression in human colonic adenocarcinoma compared with paired normal epithelium

CD91 expression in human normal colonic mucosa. As Calreticulin is a binding partner of CD91, we evaluated the expression of CD91 in 25 out of the 58 cases previously studied with Calreticulin. In normal colonic mucosa, CD91 was always expressed by epithelial cells (Fig. 3A and C). The staining was mainly cytoplasmic. CD91 was also expressed by pericryptic cells, likely myofibroblasts, and by lamina propria mononuclear cells (Fig. 3A and C). Double immunostaining followed by confocal microscopy, using anti-CD91 and $\alpha$-SMA antibodies, confirmed that $\alpha$-SMA-positive pericryptic myofibroblasts expressed CD91 (Fig. 4, upper panel). Confocal microscopy also showed that epithelial cells co-expressed CD91 and Calreticulin (Fig. 4, lower panel). Quantification of the overlay of labeling with the image processing software MetaMorph showed that the majority of 


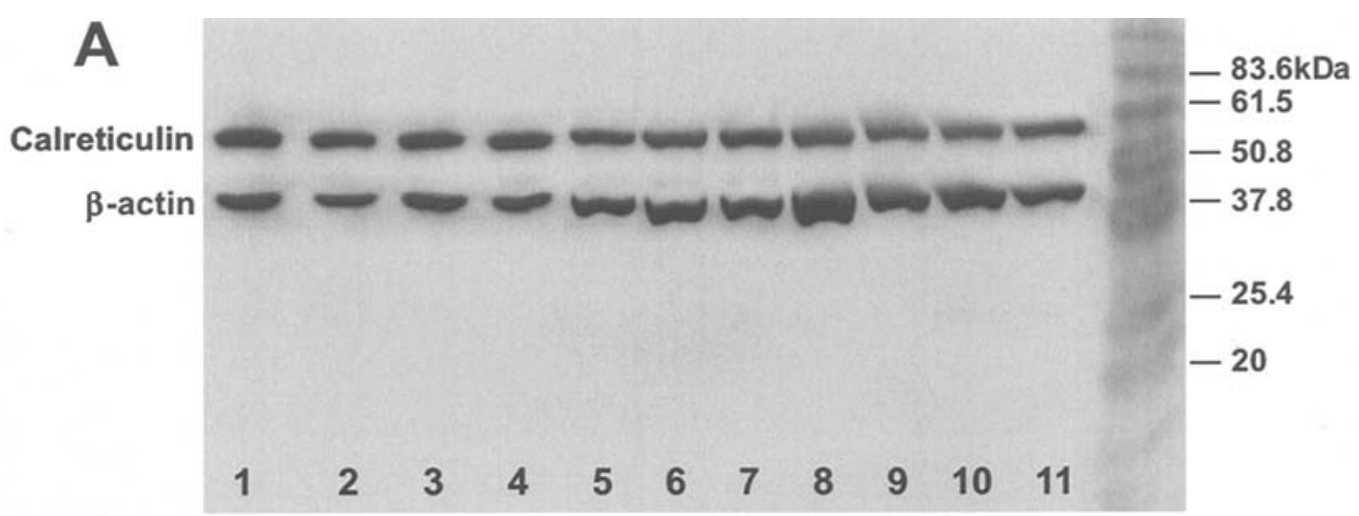

B

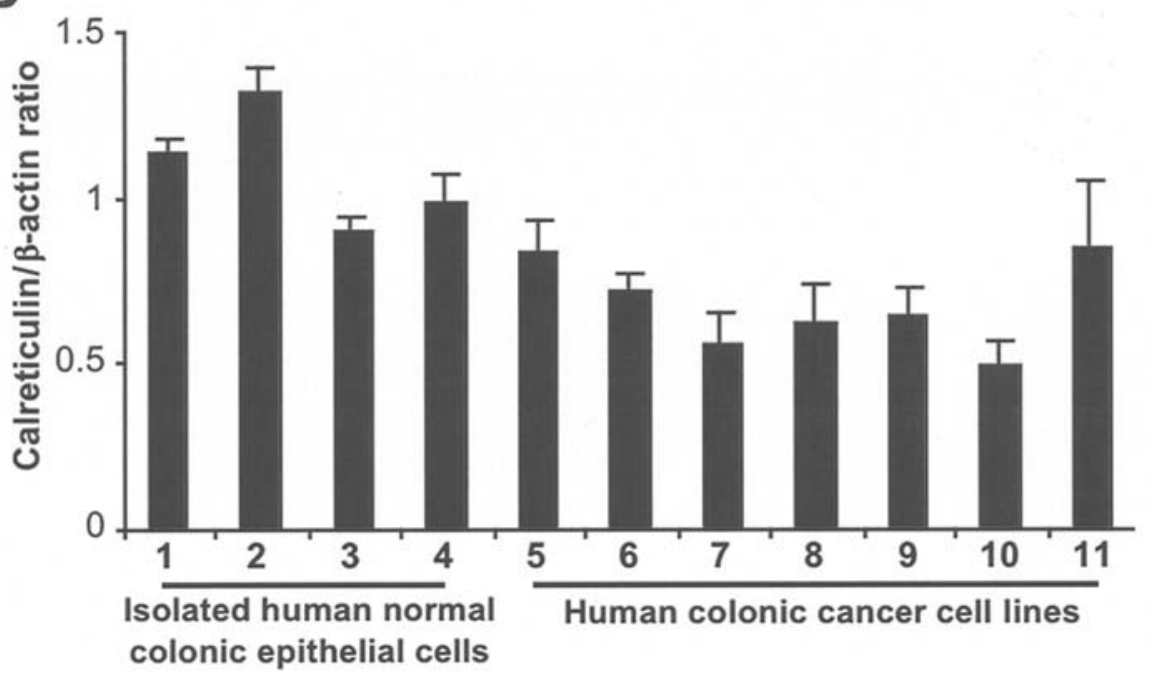

Figure 2. Immunoblot analysis of Calreticulin expression in colonic cancer cell lines compared with normal isolated colonic epithelial cells. (A) A representative immunoblot shows Calreticulin and B-actin expression in four preparations of isolated human normal colonic epithelial cells (lanes 1-4) and in seven human colonic cancer lines (lane 5, SW1116; lane 6, SW620; lane 7, SW480; lane 8, HT29; lane 9, HT29-Cl.19A; lane 10, HT29-Cl.16E; lane 11, Colo320). Molecular weight markers are shown on the right. (B) A quantitative analysis of the bands was performed with the image Quant software on three different immunoblots (mean \pm SE). A down-regulation of the Calreticulin/ß-actin ratio was observed in the seven colonic cancer cell lines tested, compared with the four preparations of isolated colonic epithelial cells.

CD91 co-localised with Calreticulin in epithelial cells $(67.5 \pm 7 \%$, mean \pm SE of 10 different regions analysed).

CD91 expression in colon adenocarcinomas. In adenocarcinomas, CD91 was down-regulated in $18 / 25$ cases $(72 \%)$ compared with paired normal epithelium, with a complete loss of expression in 4 cases (Fig. 3B). In $7 / 25$ cases (28\%), CD91 expression in tumour cells was similar to that of normal epithelial cells (Fig. 3D). In most cases, the stromal reaction within the tumour was strongly labeled. Of the 25 cases, 12 (48\%) showed a down-regulated expression of both CD91 and Calreticulin.

Association between pathological features of the tumours, and the expression of Calreticulin and/or CD91. In 25 cases, we examined a possible association between the pathological features of the tumours, i.e. localisation of the tumour (right or left colon), TNM staging, mucinous differentiation, presence of a lymphoid stromal reaction, and the expression of Calreticulin and/or CD91.

The only statistically significant association was found between Calreticulin expression and the mucinous differentiation of the tumour $(\mathrm{P}=0.006)$. The majority of mucinous adenocarcinomas $(5 / 6=83 \%)$ expressed Calreticulin at a level similar to that of paired normal epithelium, using a semiquantitative immunohistochemical scoring method. Accordingly, among the 17 cases down-regulated for Calreticulin, 16 (94\%) were devoid of mucinous component.

Moreover, when extending the analysis to the series of 58 cases immunostained with anti-Calreticulin, an association was found again between the Calreticulin expression and mucinous adenocarcinomas $(\mathrm{P}=0.002)$. The vast majority of mucinous adenocarcinomas $(12 / 14=86 \%)$ expressed Calreticulin. Accordingly, the vast majority (90\%) of tumours displaying a down-regulated expression of Calreticulin were devoid of mucinous differentiation, and are thus considered as 'classic' adenocarcinomas according to the WHO classification.

\section{Discussion}

The main findings of this study are: 1) Calreticulin expression, compared to paired normal epithelium, is down-regulated in $51.7 \%$ of human colon adenocarcinomas in a series of 58 cases; 2) the Calreticulin receptor CD91, known to be expressed by macrophages, is also expressed by normal colonic epithelial cells and pericryptic myofibroblasts; 3) both 

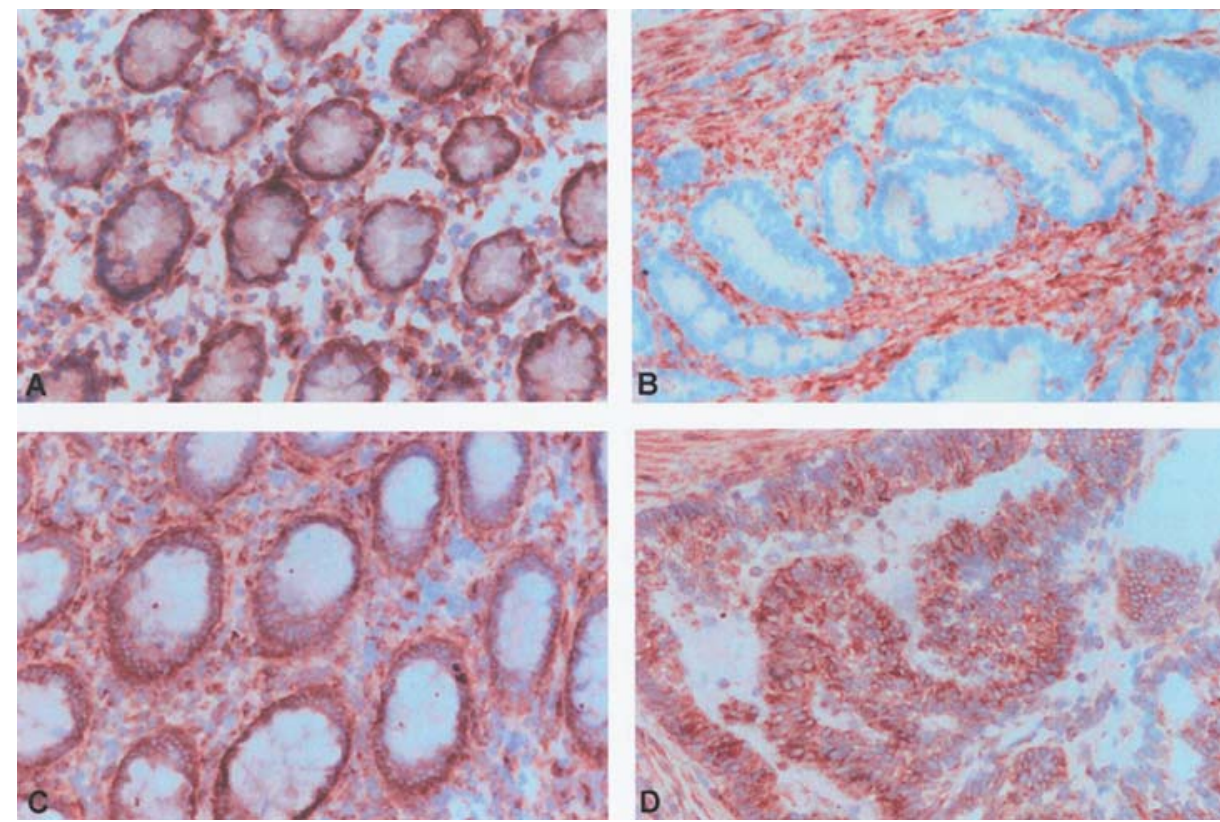

Figure 3. CD91 immunohistochemistry in human colonic adenocarcinomas compared with normal paired epithelium. Frozen sections of human adenocarcinomas and normal paired epithelium were stained with the monoclonal anti-CD91 antibody and counterstained with hematoxylin. In normal colonic mucosa (A and C), CD91 was expressed by colonic epithelial cells. CD91 was also expressed in the lamina propria by pericryptic cells, likely myofibroblats, and by mononuclear cells. In colonic adenocarcinomas (B and D), CD91 expression in neoplastic cells was either down-regulated (B) or similar to that of normal paired epithelium (D). CD91 was also expressed in the stroma of colon carcinoma. Original magnification x200.

CD91
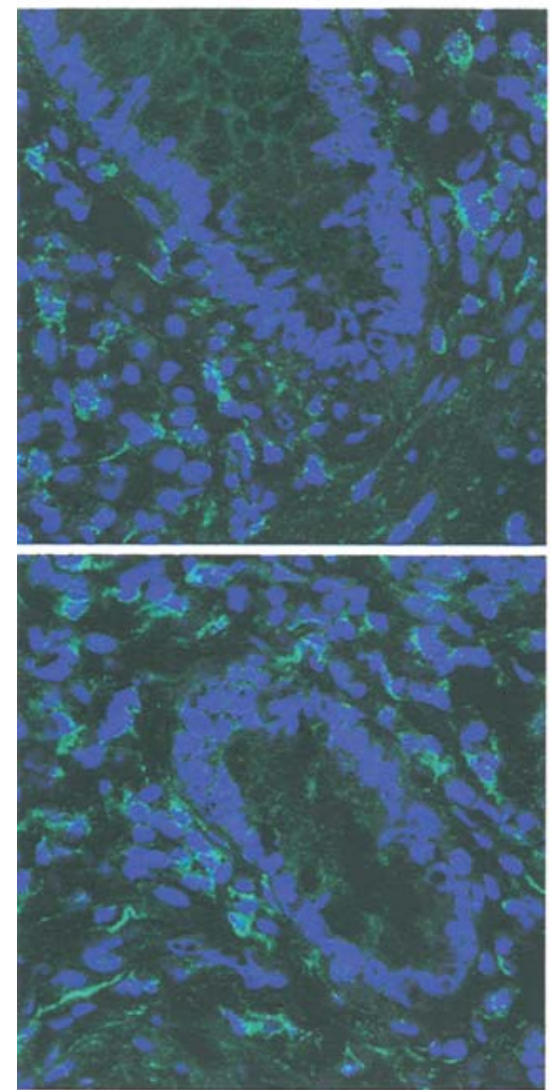

CD91
$\alpha$-SMA
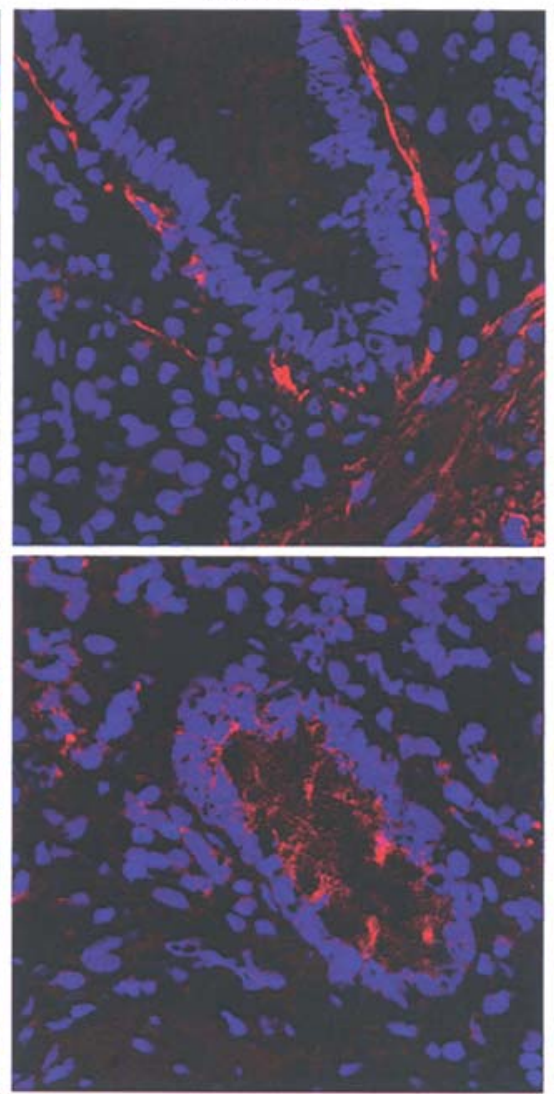

Calreticulin merge
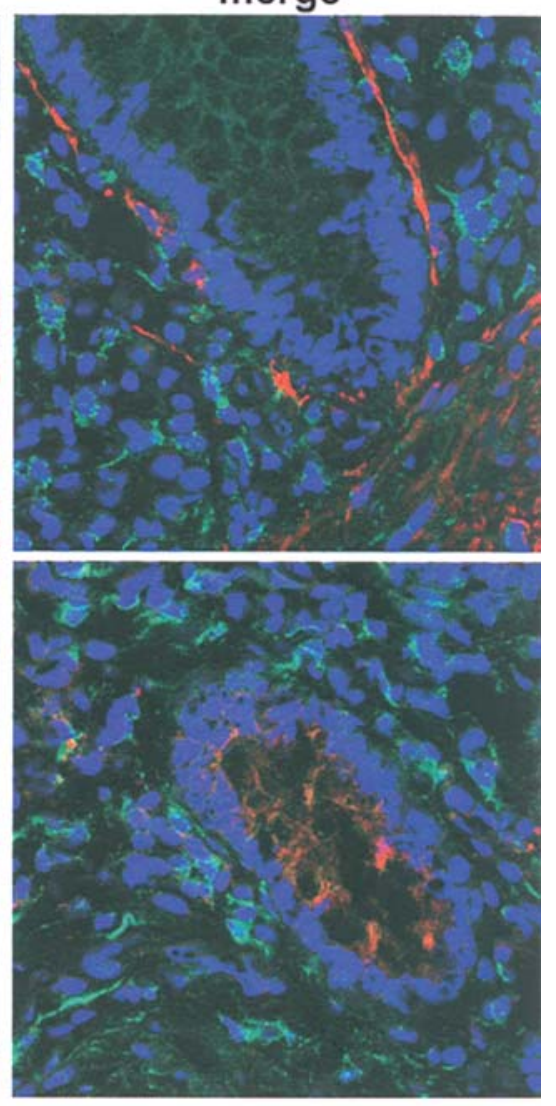

merge

Figure 4. Co-expression of CD91 and $\alpha$-SMA in human colonic myofibroblasts, and of CD91 and calreticulin in human colonic epithelial cells. Double immunostaining of CD91 (green) and $\alpha$-SMA (red) or calreticulin (red) was performed on frozen sections of human normal colon and examined by confocal microscopy as described in Materials and methods. Nuclei appear in blue. The myofibroblasts surrounding colonic crypts, scoring positive for $\alpha$-SMA, expressed CD91 (upper panel). Colonic epithelial cells, as well as some lamina propria immune cells, co-expressed CD91 and Calreticulin (lower panel). Original magnification x630. 
CD91 and Calreticulin are down-regulated in $48 \%$ of colonic adenocarcinomas, and 4) Calreticulin expression is significantly associated with the mucinous differentiation of the tumour.

Calreticulin expression in human colorectal adenocarcinomas has been studied by proteomic analysis with divergent results reported, i.e. an up-regulation (19) or a down-regulation (20). These discrepancies may be explained by the fact that these studies were restricted to whole samples of colorectal adenocarcinomas including both neoplastic and non-neoplastic cells. As Calreticulin is expressed by several cell types, analysis based on whole sample lysates may be invaluable for assessing Calreticulin expression specifically in tumour cells. Up to now, the comparative expression of Calreticulin in neoplastic and normal colonic epithelial cells by immunohistochemistry has not been addressed. The findings of our study demonstrate a down-regulation of Calreticulin expression in $51.7 \%$ of human colorectal adenocarcinomas at the protein level using a semi-quantitative immunohistochemical analysis already validated for various proteins in colon cancer $(21)$ or other neoplasms $(28,29)$. Our results were confirmed by a quantitative immunoblot analysis comparing Calreticulin expression in human isolated normal colonic epithelial cells with that of human colonic cancer cell lines. A significant down-regulation of the Calreticulin/ß-actin ratio was found in the seven colonic cancer cell lines tested, compared with the four preparations of isolated colonic epithelial cells.

This study is the first to assess the expression of Calreticulin together with its binding partner CD91 in colorectal adenocarcinomas. CD91 was identified initially as a receptor for $\alpha 2$-macroglobulin $(30,31)$, and then as the receptor for several heat-shock proteins including Calreticulin (12). CD91 is present on cells of the monocytic lineage, but also in other cell types including hepatocytes, intestinal epithelial cells, and fibroblasts (32). Recent studies have shown that CD91 complexed with Calreticulin participates in driving the clearance of epithelial apoptotic cells by other epithelial cells, so-called 'amateur phagocytes', in a model of mammary gland involution and in mammary epithelial cell lines (33). These findings suggest that Calreticulin and CD91 expressed by epithelial cells can play a role in the maintenance of tissue homeostasis. In line with this study, we extended the concept of 'amateur phagocyte' to human normal colonic epithelial cells and pericryptic $\alpha$-SMA-positive myofibroblasts, both co-expressing Calreticulin and CD91. This association could play a role in the autophagocytosis process/apoptotic cells removal, and contribute to colonic epithelial homeostasis.

Our study further demonstrates that CD91 expression was down-regulated in $72 \%$ of adenocarcinomas. In addition, $48 \%$ of adenocarcinomas showed a down-regulated expression of both CD91 and Calreticulin in neoplastic epithelial cells. These findings, showing a dramatic decrease in Calreticulin/ CD91 expression, are in line with the concept that tissue homeostasis is altered in cancer.

Finally, these findings led us to examine the possible associations between important phenotypic features of these tumours: tumour localisation, TNM staging, histological grading, presence of a lymphoid stromal reaction, mucinous differentiation, and the expression of Calreticulin and/or CD91. The only statistical association was found between the presence of Calreticulin and the mucinous differentiation of the tumour. Within the 58 cases studied, 14 were classified as mucinous adenocarcinomas, and 12 of these 14 cases expressed Calreticulin. Accordingly, among the cases down-regulated for Calreticulin expression, the vast majority (90\%) was devoid of mucinous component. Interestingly, another study has established a molecular link between Calreticulin and mucin expression (34). Using human colonic cancer cell lines and immunoprecipitation with anti-mucin and anti-Calreticulin, McCool et al (34) were able to show that Calreticulin is involved in the folding of MUC2 but not of MUC5AC. As MUC2 overexpression is a feature of mucinous carcinomas of the colon (35), and was also found in our cases of mucinous carcinomas (data not shown), these findings strongly suggest that the maintenance of Calreticulin expression is essential for chaperoning MUC2 synthesis in mucinous adenocarcinomas.

In conclusion, we show herein for the first time a molecular/ morphological association that is of importance for the understanding of the differentiation of human colonic adenocarcinomas.

\section{Acknowledgements}

This study was in part supported by two grants 'Projet Hospitalier Regional de Recherche Clinique' BRD05/10/C and BRD04/6/P. We thank Sigrid Parois and Jeanne Souchet for expert technical assistance, and the staff of the 'Photologie' Department.

\section{References}

1. Michalak M, Robert Parker JM and Opas $\mathrm{M}: \mathrm{Ca}^{2+}$ signaling and calcium binding chaperones of the endoplasmic reticulum. Cell Calcium 32: 269-278, 2002.

2. Gelebart P, Opas M and Michalak M: Calreticulin, a $\mathrm{Ca}^{2+}$ binding chaperone of the endoplasmic reticulum. Int J Biochem Cell Biol 37: 260-266, 2005 .

3. Trombetta ES: The contribution of N-glycans and their processing in the endoplasmic reticulum to glycoprotein biosynthesis. Glycobiology 13: R77-R91, 2003.

4. Coppolino MG, Woodside MJ, Demaurex N, Grinstein S, St-Arnaud R and Dedhar S: Calreticulin is essential for integrinmediated calcium signalling and cell adhesion. Nature 386 : 843-847, 1997.

5. Fadel MP, Szewczenko-Pawlikowski M, Leclerc P, Dziak E, Symonds JM, Blaschuk O, Michalak M and Opas M: Calreticulin affects beta-catenin-associated pathways. J Biol Chem 276: 27083-27089, 2001

6. Johnson S, Michalak M, Opas M and Eggleton P: The ins and outs of calreticulin: from the ER lumen to the extracellular space. Trends Cell Biol 11: 122-129, 2001.

7. Burns K, Duggan B, Atkinson EA, Famulski KS, Nemer M, Bleackley RC and Michalak M: Modulation of gene expression by calreticulin binding to the glucocorticoid receptor. Nature 367: 476-480, 1994.

8. Sadasivan B, Lehner PJ, Ortmann B, Spies T and Cresswell P: Roles for calreticulin and a novel glycoprotein, tapasin, in the interaction of MHC class I molecules with TAP. Immunity 5: 103-114, 1996.

9. Seliger B, Maeurer MJ and Ferrone S: Antigen-processing machinery breakdown and tumor growth. Immunol Today 21 : 455-464, 2000.

10. Gao B, Adhikari R, Howarth M, Nakamura K, Gold MC, Hill AB, Knee R, Michalak $M$ and Elliott T: Assembly and antigenpresenting function of MHC class I molecules in cells lacking the ER chaperone calreticulin. Immunity 16: 99-109, 2002. 
11. Culina S, Lauvau G, Gubler B and van Endert PM: Calreticulin promotes folding of functional human leukocyte antigen class I molecules in vitro. J Biol Chem 279: 54210-54215, 2004.

12. Basu S, Binder RJ, Ramalingam T and Srivastava PK: CD91 is a common receptor for heat shock proteins gp96, hsp90, hsp70, and calreticulin. Immunity 14: 303-313, 2001.

13. Binder RJ, Karimeddini D and Srivastava PK: Adjuvanticity of alpha 2-macroglobulin, an independent ligand for the heat shock protein receptor CD91. J Immunol 166: 496-4972, 2001.

14. Henson PM, Bratton DL and Fadok VA: Apoptotic cell removal. Curr Biol 11: 795-805, 2001.

15. Vandivier RW, Ogden CA, Fadok VA, Hoffmann PR, Brown KK, Botto M, Walport MJ, Fisher JH, Henson PM and Greene KE: Role of surfactant proteins A, D, and C1q in the clearance of apoptotic cells in vivo and in vitro: calreticulin and CD91 as a common collectin receptor complex. J Immunol 169: 3978-3986, 2002.

16. Facciponte JG, MacDonald IJ, Wang XY, Kim H, Manjili MH and Subjeck JR: Heat shock proteins and scavenger receptors: role in adaptive immune responses. Immunol Invest 34: 325-342, 2005.

17. Gardai SJ, McPhillips KA, Frasch SC, Janssen WJ, Starefeldt A, Murphy-Ullrich JE, Bratton DL, Oldenborg PA, Michalak M and Henson PM: Cell-surface calreticulin initiates clearance of viable or apoptotic cells through transactivation of LRP on the phagocyte. Cell 123: 332-334, 2005.

18. Cole AR, Ji H and Simpson RJ: Proteomic analysis of colonic crypts from normal, multiple intestinal neoplasia and p53-null mice: a comparison with colonic polyps. Electrophoresis 21: 1772-1781, 2000

19. Brunagel G, Shah U, Schoen RE and Getzenberg RH: Identification of calreticulin as a nuclear matrix protein associated with human colon cancer. J Cell Biochem 89: 238-243, 2003.

20. Alfonso P, Nunez A, Madoz-Gurpide J, Lombardia L, Sanchez L and Casal JI: Proteomic expression analysis of colorectal cancer by two-dimensional differential gel electrophoresis. Proteomics 5: 2602-2611, 2005.

21. Blanchot-Jossic F, Jarry A, Masson D, Bach-Ngohou K, Paineau J, Denis MG, Laboisse CL and Mosnier JF: Upregulated expression of ADAM17 in human colon carcinoma: co-expression with EGFR in neoplastic and endothelial cells. J Pathol 207: 156-163, 2005.

22. Jarry A, Bach-Ngohou K, Masson D, Dejoie T, Lehur PA, Mosnier JF, Denis MG and Laboisse CL: Human colonic myocytes are involved in postischemic inflammation through ADAM17-dependent TNFalpha production. Br J Pharmacol 147: 64-72, 2006.

23. Branka JE, Vallette G, Jarry A, Bou-Hanna C, Lemarre P, Van PN and Laboisse CL: Early functional effects of Clostridium difficile toxin A on human colonocytes. Gastroenterology 112: 1887-1894, 1997.
24. Fogh $\mathrm{J}$ and Trempe G: Human Tumor Cells in vitro: New Human Tumor Cell Lines. Fogh J (ed). Plenum Publishing Corp., New York, pp115-141, 1989.

25. Augeron $\mathrm{C}$ and Laboisse CL: Emergence of permanently differentiated cell clones in a human colonic cancer cell line in culture after treatment with sodium butyrate. Cancer Res 47: 396-3969, 1984.

26. Leibovitz A, Stinson JC, Mc Combs WB III, McCoy CE, Mazur KC and Mabry ND: Classification of human colorectal adenocarcinoma cell lines. Cancer Res 36: 4562-4569, 1976.

27. Quinn LA, Moore GE, Morgan Rt and Woods LK: Cell lines from human colon carcinoma with unusual cell products, double minutes, and homogeneously staining regions. Cancer Res 39: 4914-4924, 1979

28. Nakamura H, Takeshima H, Makino $\mathrm{K}$ and Kuratsu J: C-kit expression in germinoma: an immunohistochemistry-based study. J Neurooncol 75: 163-167, 2005.

29. Shabnam MS, Srinivasan R, Wali A, Majumdar S, Joshi K and Behera D: Expression of p53 protein and the apoptotic regulatory molecules Bcl-2, Bcl-XL, and Bax in locally advanced squamous cell carcinoma of the lung. Lung Cancer 45: 181-188, 2004.

30. Moestrup SK and Gliemann J: Purification of the rat hepatic alpha 2-macroglobulin receptor as an approximately $440-\mathrm{kDa}$ single chain protein. J Biol Chem 264: 15574-15577, 1989.

31. Ashcom JD, Tiller SE, Dickerson K, Cravens JL, Argraves WS and Strickland DK: The human alpha 2-macroglobulin receptor: identification of a $420-\mathrm{kD}$ cell surface glycoprotein specific for the activated conformation of alpha 2-macroglobulin. J Cell Biol 110: 1041-1048, 1990.

32. Moestrup SK, Gliemann J and Pallesen G: Distribution of the alpha 2-macroglobulin receptor/low density lipoprotein receptorrelated protein in human tissues. Cell Tissue Res 269: 375-382, 1992.

33. Monks J, Rosner D, Geske FJ, Lehman L, Hanson L, Neville MC and Fadok VA: Epithelial cells as phagocytes: apoptotic epithelial cells are engulfed by mammary alveolar epithelial cells and repress inflammatory mediator release. Cell Death Differ 12: 107-114, 2005.

34. McCool DJ, Okada Y, Forstner JF and Forstner GG: Roles of calreticulin and calnexin during mucin synthesis in LS180 and HT29/A1human colonic adenocarcinoma cells. Biochem J 341: 593-600, 1999.

35. Hanski C, Hofmeier M, Schmitt-Graff A, Riede E, Hanski ML, Borchard F, Sieber E, Niedobitek F, Foss HD, Stein H and Riecken EO: Overexpression or ectopic expression of MUC2 is the common property of mucinous carcinomas of the colon, pancreas, breast, and ovary. J Pathol 182: 385-391, 1997. 